\title{
Polymerization of Bicyclic Acetals. II. * Steric Course in the Cationic Polymerization of 6,8-Dioxabicyclo[3.2.1]octane
}

\author{
Masahiko OKada, Hiroshi Sumitomo, and Yasuo Hibino \\ Faculty of Agriculture, Nagoya University, Chikusa, Nagoya, Japan.
}

(Received December 25, 1973)

\begin{abstract}
The polymerization of 6,8-dioxabicyclo[3.2.1]octane (DBO) was carried out in toluene, methylene chloride, and 1-nitropropane over the wide range of temperatures from -78 to $30^{\circ} \mathrm{C}$. Boron trifluoride etherate was used as an initiator. The stereoregularity of the polymer obtained was evaluated from the relative intensities of the two NMR peaks appearing at $\tau 5.15$ and 5.60, which were due to the equatorial and axial acetal protons of the tetrahydropyran ring, respectively. The EQ-H\% of the polymer, defined as the percent of the equatorial acetal protons to the total acetal protons, decreases from nearly 100 to $70-80$ depending on the solvents used with the rise in the polymerization temperature. On the basis of these results, it is concluded that the cationic polymerization of DBO proceeds at lower temperatures through an $\mathrm{S}_{\mathrm{N}} 2$ mechanism involving the reverse side attachment of the monomer to the acetal carbon atom of a propagating cyclic trialkyloxonium ion to afford a stereoregular polymer. At higher temperatures and in polar solvents, the participation of a carboxonium ion derived from the cyclic trialkyloxonium ion in the propagation reaction and/or the competitive oxonium ion exchange reaction at the propagating chain end becomes significant, thus causing the loss of stereospecificity of the polymerization.

KEY WORDS 6,8-Dioxabicyclo[3.2.1]octane / Cationic Polymerization / Stereochemistry / Propagating Species / Oxonium Ion / Carboxonium Ion /
\end{abstract}

In a previous short communication, ${ }^{1}$ we reported that 6,8-dioxabicyclo[3.2.1] Joctane (hereafter referred to as DBO) underwent cationic polymerization to yield stereoregular high-molecular-weight polymers at $-78^{\circ} \mathrm{C}$ and stereoirregular low-molecular-weight polymers at $0^{\circ} \mathrm{C}$. This finding prompted us to elucidate what factors control the stereospecific course of the cationic polymerization of DBO. In this paper, we wish to present the experimental results concerning the effects of solvents and polymerization temperature on the stereoregularity of the polymer and to discuss the propagation mechanism in the cationic polymerization of DBO.

\section{EXPERIMENTAL}

Materials

DBO was prepared from 3,4-dihydro-2H-pyran2-carboxyaldehyde (acrolein dimer) by the method of Sweet and Brown. ${ }^{2}$ It was dissolved in meth-

* Part 1, ref 1 . ylene chloride and the solution was stirred over calcium hydride for several days. The monomer was then distilled under reduced pressure: bp $68^{\circ} \mathrm{C} / 38 \mathrm{mmHg}$ (lit. ${ }^{2} 65^{\circ} \mathrm{C} / 30 \mathrm{mmHg}$ ), mp 47$49^{\circ} \mathrm{C}$ (lit. ${ }^{2} 47-49^{\circ} \mathrm{C}$ ).

Toluene was washed successively with concd sulfuric acid, water, dil aqueous sodium hydroxide, and water again and then refluxed over, and distilled with, sodium metal. Methylene chloride was purified by washing with dil aqueous sodium carbonate and water, followed by refluxing over, and distilling with, phosphorus pentoxide. 1-Nitropropane was stirred over calcium hydride for several days and distilled under reduced pressure.

Boron trifluoride etherate was distilled just before use.

\section{Polymerization Procedure}

The polymerization was carried out in an evacuated sealed tube, or in an Erlenmeyer flask equipped with a three-way stop cock under 
nitrogen. In the former case, the polymerization was terminated by the addition of a small amount of trimethylamine dissolved in methylene chloride, and then the reaction mixture was poured into a large volume of methanol to precipitate the polymer. It was reprecipitated by using chloroform-methanol as a solventprecipitant pair. The combined methanol solution was concentrated under reduced pressure to $40-50 \mathrm{ml}$, to which an equal volume of water was added to recover the methanol soluble polymer.

In the latter case (the polymerization in an Erlenmeyer flask), a small portion of a reaction mixture was taken out at specified intervals by a syringe through a three-way stopcock under a stream of dry nitrogen, and immediately injected into a cold solution of trimethylamine in methylene chloride to interrupt the polymerization. The resulting mixture was then subjected to gas chromatography for the determination of the conversion.

\section{Characterization}

NMR spectra of the polymers were recorded on Japan Electronics Model JNM-4H-100 and JNM-MH-100 instruments working at $100 \mathrm{MHz}$ in deuteriochloroform solutions at room temperature. Tetramethylsilane was used as an internal standard.

The number average molecular weight of the polymer was determined with a Hewlett-Packard vapor-pressure osmometer Model 302 using benzene solutions at $37^{\circ} \mathrm{C}$.

\section{RESULTS}

As reported in a previous paper, ${ }^{1}$ the polymerization of DBO initiated with boron trifluoride etherate yields a large amount of a methanolsoluble polymer together with a methanolinsoluble polymer at $0^{\circ} \mathrm{C}$ irrespective of the solvents used, while it affords only a methanolinsoluble polymer at $-78^{\circ} \mathrm{C}$.

Figure 1 shows the NMR spectra of the methanol-insoluble polymers prepared at (A) $0^{\circ} \mathrm{C}$ and $(\mathrm{B})-78^{\circ} \mathrm{C}$. From the relative peak intensities of the spectrum $\mathrm{A}$, the peaks at $\tau 5.15$ and 5.60 can both be assigned to the acetal protons, while the complicated peaks ranging

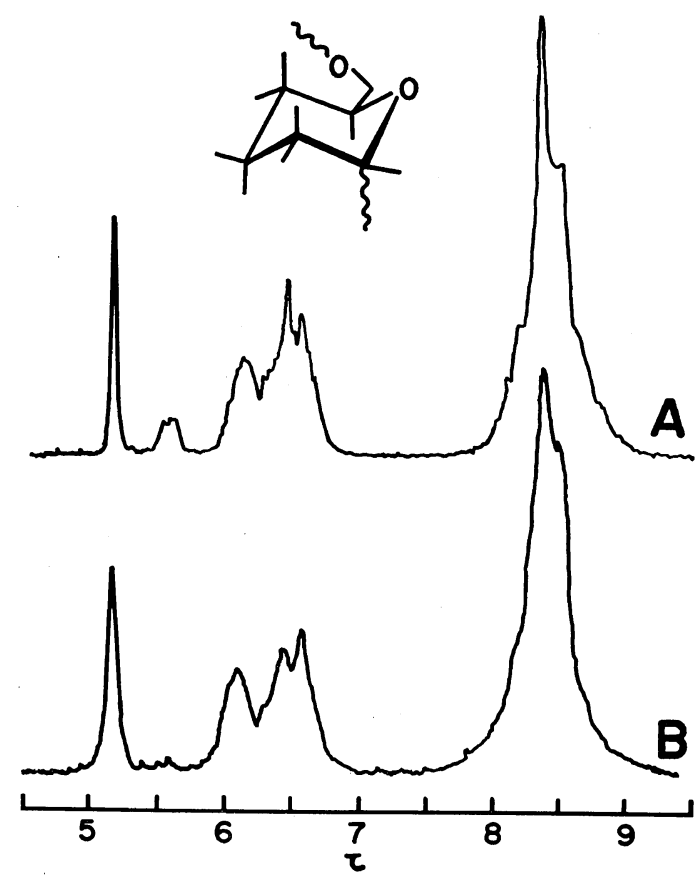

Figure 1. NMR spectra of poly-6,8-dioxabicyclo[3.2.1]octane prepared at $(\mathrm{A}) 0^{\circ} \mathrm{C}$ and $(\mathrm{B})-78^{\circ} \mathrm{C}$ : Solvent, $\mathrm{CDCl}_{3}$; concn, ca. $5 \%$; room temp; 100 $\mathrm{MHz}$.

from $\tau 5.9$ to 6.8 can be assigned to the methylene and methine protons adjacent to one oxygen atom, and the strong broad peak in the region of $\tau 7.5$ to 9.0 to the methylene protons of the tetrahydropyran ring. Equatorial protons in the cyclohexane and pyranose rings generally give rise to resonance at a lower field than their axial counterparts. ${ }^{3}$ This relationship appears valid in the present case, and hence the peaks at $\tau 5.15$ and 5.60 are assignable to the equatorial and axial protons of the tetrahydropyran ring, respectively. This assignment is justified by the larger width of the latter peak, because the coupling constant between this axial acetal proton and its vicinal axial proton is larger than the corresponding value of the equatorial acetal proton. The NMR spectrum of the methanolsoluble part is essentially the same as that of the methanol-insoluble part, indicating that the difference in solubility originates from the molecular weight, not from the stereo-irregularity. In the spectrum $B$, the peak at $\tau 5.60$ almost disappears, and there is practically only one 
peak at $\tau 5.15$. This is clearly indicative of the higher stereoregularity of the polymer prepared at $-78^{\circ} \mathrm{C}$.

In the foregoing assignment of the NMR spectra, it is assumed that the $\mathrm{C}_{5}-\mathrm{O}_{6}$ bond of DBO monomer is cleaved when it undergoes ring-opening polymerization. The cleavages of the $\mathrm{O}_{6}-\mathrm{C}_{7}$ and $\mathrm{C}_{1}-\mathrm{O}_{8}$ bonds can be ruled out on the basis of the studies on the hydrolysis of cyclic acetals ${ }^{4}$ and the NMR analysis of the microstructure of the copolymer derived from 1,3-dioxolane and styrene. ${ }^{5}$ The possibility of the $\mathrm{C}_{5}-\mathrm{O}_{8}$ bond scission could be safely excluded for the following reasons:

1) 2-Hydroxymethyl-3,4-dihydro-2H-pyran, a precursor of $\mathrm{DBO}$, polymerizes on standing at room temperature in the presence of a small amount of hydrogen chloride. $n$<smiles>CC1CCCC(COC(C)(C)C)O1</smiles>

The NMR and IR spectra of the polymer thus obtained are essentially the same as those of the polymer formed in the polymerization of $\mathrm{DBO}$ at $0^{\circ} \mathrm{C}$. If $\mathrm{DBO}$ polymerized through the $\mathrm{C}_{5}-\mathrm{O}_{8}$ bond scission, the resulting polymer would have a structure consisting of oxacycloheptane rings bridged by an oxygen atom at the 2 and 6-positions.

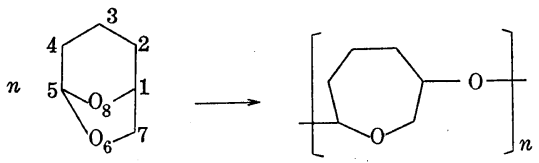

Therefore, this polymer could be differentiated spectroscopically from the polymer derived from 2-hydroxymethyl-3,4-dihydro-2H-pyran, which consists of alternating tetrahydropyran and oxymethylene units.

2) It is reported by Clasper and Brown ${ }^{6}$ that the hydrogenolysis of DBO by a mixture of lithium aluminum hydride and aluminum chloride gives quantitatively 2-hydroxymethyl tetrahydropyran resulting from the $\mathrm{C}_{5}-\mathrm{O}_{6}$ bond scission.

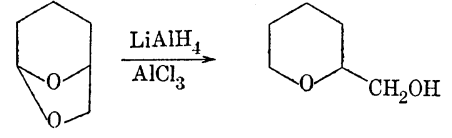

These facts strongly substantiate the exclusive cleavage of the $\mathrm{C}_{5}-\mathrm{O}_{6}$ bond of $\mathrm{DBO}$ monomer in its ring-opening polymerization.

As described above, the stereoregularity of the polymer can be estimated from the relative intensities of the two NMR peaks appearing at $\tau 5.15$ and 5.60, which are due to the equatorial and axial acetal protons, respectively. Here we use as a measure of the stereoregularity EQ$\mathrm{H} \%$, which is defined as the percent of the equatorial acetal protons relative to the total acetal protons.

Figure 2 illustrates the temperature dependence of $\mathrm{EQ}-\mathrm{H} \%$ 's of the polymers obtained in (A) toluene, (B) methylene chloride, and (C) 1-nitropropane. No significant difference is observed at $-78^{\circ} \mathrm{C}$ and at the temperatures above $0^{\circ} \mathrm{C}$ among the $\mathrm{EQ}-\mathrm{H} \%$ 's of the polymers from the three series of experiments with different solvents. However, in the temperature range from -40 to $-20^{\circ} \mathrm{C}$, the $\mathrm{EQ}-\mathrm{H} \%$ 's of the polymers from these series decrease in the order: toluene $>$
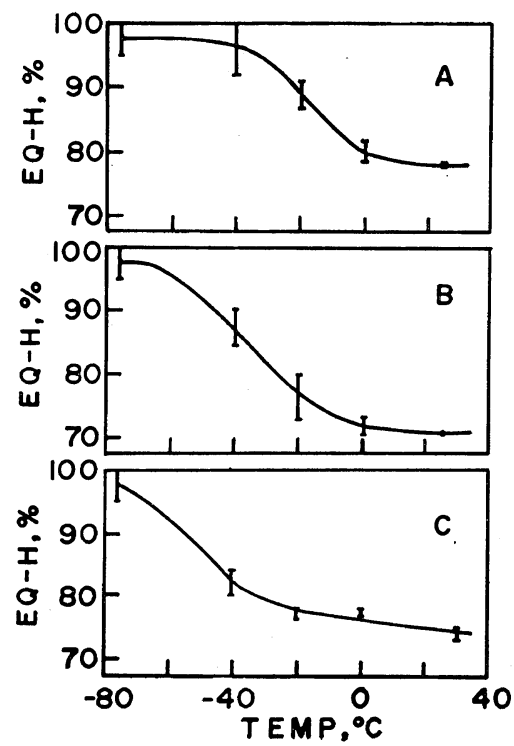

Figure 2. Temperature dependence of $\mathrm{EQ}-\mathrm{H} \%$ : Solvent, A, toluene; B, methylene chloride; C, 1nitropropane. 
Polymerization of 6,8-Dioxabicyclo[3.2.1]octane

Table I. Polymerization of 6,8-dioxabicyclo[3.2.1]octane ${ }^{\mathrm{a}}$

\begin{tabular}{lccccccc}
\hline No. & {$[\mathrm{M}]_{0}, \mathrm{~mol} / l$} & {$[\mathrm{I}]_{0}, \mathrm{mmol} / l$} & Time, day & {$[\mathrm{M}]_{\mathrm{r}}{ }^{\mathrm{b}}, \mathrm{mol} / l$} & Convn, $\%$ & $\mathrm{EQ}-\mathrm{H}, \%$ & $\boldsymbol{M}_{n} \mathrm{c}^{\mathrm{c}}$ \\
\hline e-8 & 2.06 & 20.7 & 1 & 1.45 & 29.9 & 91 & 2900 \\
e-9 & 2.06 & 20.7 & 3 & 0.291 & 85.9 & 86 & 4300 \\
e-12 & 1.99 & 25.0 & 2 & 0.108 & 94.6 & 84 & 5400 \\
e-13 & 1.99 & 25.0 & 7 & 0.086 & 95.7 & 77 & 5600 \\
e-14 & 1.99 & 25.0 & 9 & 0.086 & 95.7 & 76 & 4300 \\
e-15 & 1.99 & 25.0 & 12 & 0.086 & 95.7 & 75 & 4500
\end{tabular}

a Initiator, boron trifluoride etherate; solvent, methylene chloride; temp, $-40^{\circ} \mathrm{C}$.

b Unreacted monomer concentration.

c Unfractionated polymer.

methylene chloride $>1$-nitropropane. The temperature where significant change in the EQ$\mathrm{H} \%$ of the polymer occurs decreases with the increase in the polarity of the solvent. The indicated range of each plot in Figure 2 denotes the upper and lower values observed for two or more runs.

Table I shows the results of the polymerization of DBO carried out in methylene chloride at $-40^{\circ} \mathrm{C}$. It appears that the $\mathrm{EQ}-\mathrm{H} \%$ of the isolated polymers, which include both methanolsoluble and methanol-insoluble parts, decreases very slightly with conversions (No. e-8-e-12), but when the reaction mixture was allowed to stand at $-40^{\circ} \mathrm{C}$ for a prolonged time after it had arrived near the equilibrium conversion, the $\mathrm{EQ}-\mathrm{H} \%$ of the polymer decreased appreciably to a constant value of 75-77\%. (No. e-12e-15).

Figure 3 demonstrates the conversion dependence of the $\mathrm{EQ}-\mathrm{H} \%$ of the polymers obtained in the polymerization in toluene at $0^{\circ} \mathrm{C}$. It is noteworthy that the EQ-H\%'s are nearly constant throughout the polymerization, at least in this conversion range.

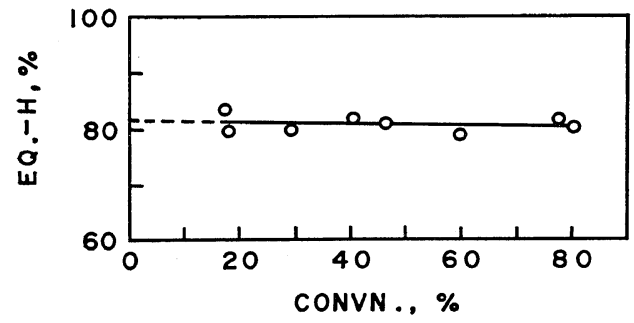

Figure 3. Conversion dependence of $\mathrm{EQ}-\mathrm{H} \%$ : Solvent, toluene; temp, $0^{\circ} \mathrm{C}$.

\section{DISCUSSION}

On the basis of the foregoing experimental results, we will discuss the mechanism of the propagation in the cationic polymerization of DBO. Let us first assume that the growing chain end is a cyclic trialkyloxonium ion $\mathbf{I}$, as depicted in Scheme 1. DBO monomer approaches from the opposite side of the positively charged oxygen atom to the acetal carbon atom of the terminal unit (broken arrow a) and cleaves the carbon-oxygen bond of the oxonium ion by an $S_{\mathrm{N}} 2$-type reaction. This process results in the regeneration of the cyclic trialkyloxonium ion $\mathbf{I}$ with its degree of polymerization increased by one, but this oxonium ion is represented anew by the structure II in order to show the configuration of the penultimate tetrahydropyran ring 2 produced in this propagation step. The structure II might be converted to the structure III by the flipping of the tetrahydropyran ring 2 , but this interconversion seems less likely to take place, at least at lower temperatures, because it was found that the polymers prepared at $-78^{\circ} \mathrm{C}$ had exclusively equatorial acetal protons, that is, the configuration of almost all the tetrahydropyran rings in the polymer chain was the same as that of the tetrahydropyran ring 2 in the structure II. The addition of the monomer to the acetal carbon atom of the propagating ion I from the same side of the positively charged oxygen atom would lead to the structure VI (the structure $\mathbf{V}$ which possesses two bulky substituents both in the axial positions is energetically highly unfavorable and can be neglected), but this type of reaction appears improbable because of the severe steric hindrance. There- 


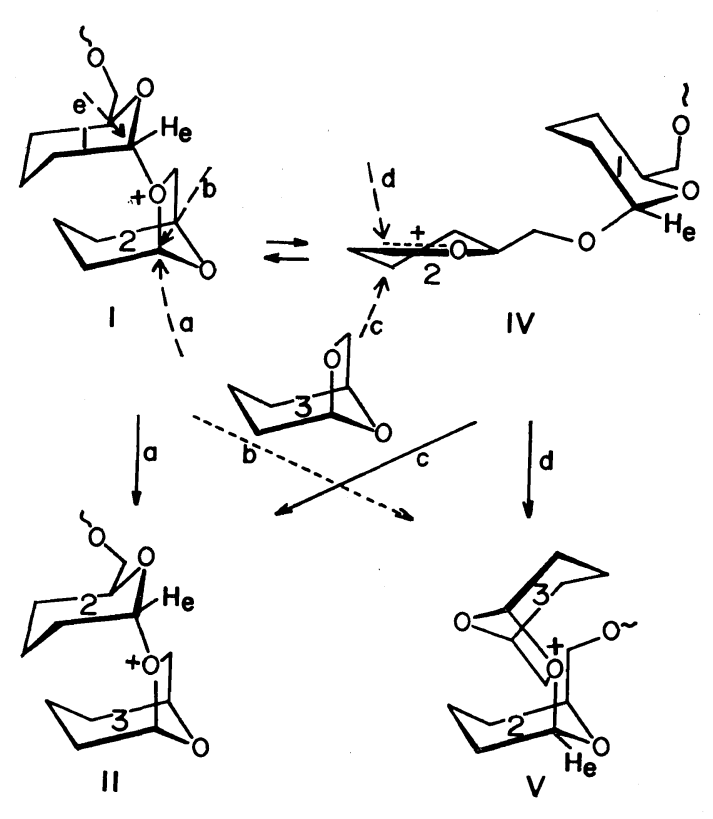

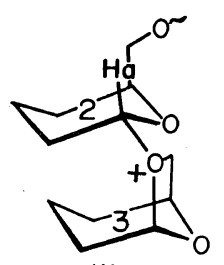

III

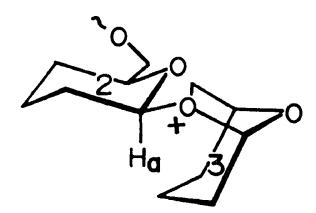

VI
Scheme 1.

fore, the fact that the stereoregular polymers possessing acetal hydrogens in the equatorial positions are obtained in the polymerization at $-78^{\circ} \mathrm{C}$ is reasonably explained in terms of the $\mathrm{S}_{\mathrm{N}} 2$ mechanism involving the reverse side attack of the monomer to the acetal carbon atom of the cyclic trialkyloxonium ion $\mathbf{I}$.

As illustrated in Figure 2, the higher the polymerization temperature and/or the polarity of the solvent, the lower the $\mathrm{EQ}-\mathrm{H} \%$ of the polymer obtained; in other words, the fraction of the structural unit having its acetal hydrogen in the axial position increases in the polymer chain. There are two possible ways to interpret this phenomenon. The first is the concept that the growing chain end in the cationic polymerization of DBO consists of the cyclic trialkyloxonium ion $I$ and the carboxonium ion IV, the latter of which is derived from the former by monomolecular ring-opening reaction. This concept was proposed by Schuerch and his coworker ${ }^{7}$ to explain the observed decrease in the specific rotation of the polymers obtained at higher temperatures in the cationic polymerization of 1, 6-anhydro-2, 3, 4-tri- $O$-benzyl-( $\beta$ )-D-mannopyranose. The configuration of the positively charged carbon atom of the carboxonium ion IV is nearly flat, and hence the addition of DBO monomer to this carbon atom would be possible from either side of the plane of the carboxonium ion IV. If the monomer attacks the carbon atom from below (broken arrow c), the structure II is formed, and conversely if the monomer adds from above (broken arrow d), the structure VI is produced through the structure $\mathbf{V}$. The cyclic trialkyloxonium ion $\mathbf{I}$ and the carboxonium IV are probably in equilibrium. The former, as described above, reacts stereospecifically to afford the structure II at one rate, and the latter nonstereospecifically to yield the structures II and VI at another rate. Therefore, the results in Figure 2 can be rationalized by assuming that the contribution of the carboxonium ion IV to the formation of the polymer becomes greater with the rise in polymerization temperature and/or the polarity of the solvent.

The second explanation for the formation of the stereoirregular polymer is that regardless of the polymerization temperature and the solvent, the propagation is always an $\mathrm{S}_{\mathrm{N}} 2$-type reaction in which the monomer attacks the oxonium ion from the back to give a structural unit having its acetal proton in the equatorial position; the subsequent inversion of this configuration of the acetal carbon atom caused by some side reactions would be responsible for the stereoirregularity of the polymer chain. Side reactions which reduce the stereoregularity of the polymer chain may include oxonium ion-exchange reaction at the growing chain end and acetal-exchange reaction involving the growing chain end and the polymer chain.

When DBO monomer (marked 3 for convenience) attacks the positively charged acetal carbon atom of the penultimate unit 1 , instead of the terminal unit 2 of the propagating cyclic trialkyloxonium ion $\mathrm{I}$ by an $\mathrm{S}_{\mathrm{N}} 2$ mechanism (broken arrow e in Scheme 1), the terminal unit 2 is replaced by the new monomeric unit 3 and 
concomitantly the configuration of the acetal carbon atom of the penultimate tetrahydropyran ring 1 is inverted. This oxonium ion exchange reaction is expected to occur less frequently at lower temperatures because the addition of the monomer to the acetal carbon atom of the penultimate tetrahydropyran ring 1 seems to require a higher activation energy than that for the addition to the acetal carbon of the terminal unit 2, which is strained and hence more reactive due to its bicyclic structure.

The reaction of the cyclic trialkyloxonium ion I with an oxygen atom in a polymer chain produces an acyclic trialkyloxonium ion VII, as illustrated in Scheme 2. When DBO monomer attacks the acetal carbon atom of the tetrahydropyran ring 1 of this acyclic trialkyloxonium ion VII (broken arrow $f$ ) and cleaves the bond between the acetal carbon atom and the positively charged oxygen atom, a cyclic trialkyloxonium ion and a polymer chain will be formed. The configuration of the penultimate tetrahydropyran ring 1 of the resulting oxonium ion would be the same as that of the tetrahydropyran ring 2 of the structure VI in Scheme 1; the acetal hydrogen lies in the axial position. The attack of the oxygen atom of the oxymethylene group attached to the tetrahydropyran ring 1 on the acetal carbon atom of the same tetrahydropyran ring 1 may also take place (broken arrow $\mathrm{g}$ ). This reaction, however, regenerates the cyclic trialkyloxonium ion $\mathbf{I}$ and a polymer chain, and does not cause any alteration in the overall configuration of the polymer chains which have participated in the formation of the acyclic oxonium ion VII.

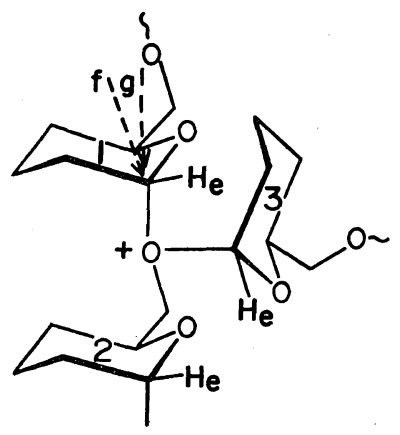

VII

Scheme 2.
Whether the cyclic trialkyloxonium ion $\mathbf{I}$ reacts with the monomer or the polymer depends on their concentrations and reactivities. There is no significant difference in the basicities of the monomer $\left(p K_{\mathrm{b}}=6.94\right)$ and the polymer $\left(p K_{\mathrm{b}}=\right.$ 6.97) determined from the shift values of the infrared $\mathrm{O}-\mathrm{D}$ stretching band of methanol- $d$ by Gordy's method. ${ }^{8}$ Accordingly, the nucleophilicities of the monomer and the polymer toward the cyclic trialkyl oxonium ion $\mathbf{I}$ are alike or, in view of the steric requirement for the reaction, the monomer may surpass the polymer in the nucleophilic reactivity. Therefore, in the early stage of the polymerization where the concentration of the polymer is low, the inversion of the acetal carbon of the tetrahydropyran ring through the acyclic trialkyloxonium ion VII seems of little importance.

In addition to these side reactions which depress the stereoregularity of the polymer chain, there may be another cause for the formation of the stereoirregular polymer. This would be the flipping of the tetrahydropyran ring. As already mentioned, the flipping of the tetrahydropyran ring 2 of the structure II to the structure III in Scheme 1 seems unlikely at $-78^{\circ} \mathrm{C}$. Furthermore, the EQ-H $\%$ 's of a polymer determined by NMR at different temperatures (room temperature and $60^{\circ} \mathrm{C}$ in deuteriochloroform) were the same values within the accuracy of the measurement. This is an indication that the flipping of the tetrahydropyran rings in the dead polymer chain does not occur appreciably in this temperature range. Nevertheless, there remains some uncertainty in precluding the flipping of the penultimate tetrahydropyran ring 2 in the structure II, particularly in the polymerization at higher temperatures and in polar solvents, as the energy difference between the structures II and III does not appear so great. In order to settle this problem, the configuration of the other asymmetric carbon atom of the tetrahydropyran ring in the polymer chain must be clarified. Unfortunately, however, the NMR signals of the methine proton of this carbon atom are overlapped badly by the signals due to the methylene protons adjacent to the oxygen atom, and quantitative information about the configuration of the asymmetric carbon atom can not be obtained.

As is shown in Figure 3, the EQ- $\mathrm{H} \%$ 's of 
the polymers obtained in the polymerization in toluene at $0^{\circ} \mathrm{C}$ are nearly constant over the conversion range from 10 to $80 \%$. This is interpretable by assuming the presence of the carboxonium ion IV as a propagating species, or alternatively by the oxonium ion-exchange reaction at the growing chain end which occurs concurrently with the propagation through the trialkyloxonium ion. Both the propagation through the carboxonium ion IV and the oxonium ion exchange reaction can provide a polymer chain possessing equatorial and axial acetal hydrogens in a constant proportion over a wide range of conversion. Which of these two possibilities is correct can not be definitely decided at present, but it would be more plausible to ascribe the constancy of the $\mathrm{EQ}-\mathrm{H} \%$ to the former, namely, the carbonium ionic character of the propagating species, for the following reason: generally cyclic ethers such as tetrahydrofuran and 3,3-bis-chloromethyl oxacyclobutane which propagate through cyclic trialkyloxonium ions do not copolymerize cationically with vinyl monomers. ${ }^{9-11}$ It was found, however, that DBO copolymerized readily with styrene in methylene chloride at $0^{\circ} \mathrm{C}$ to afford a random copolymer, while it did not at $-78^{\circ} \mathrm{C}$. $^{12}$

The inversion of the configuration through the acyclic oxonium ion VII must be of minor importance in this case, because if this reaction predominated, the $\mathrm{EQ}-\mathrm{H} \%$ of the polymer should decrease with the increase in conversion.

Table I indicates that the EQ-H\%'s of the polymers prepared in the polymerization in methylene chloride at $-40^{\circ} \mathrm{C}$ decreased to a constant value when the reaction mixture was allowed to stand for a long period after the polymerization had reached the equilibrium conversion. This behavior may suggest the inversion of the configuration of the polymer chain through the acyclic trialkyloxonium ion VII. The occurrence of this type of reaction is further confirmed by the following depolymerization experiment. When a methylene chloride solution of a stereoregular polymer $(\mathrm{EQ}-\mathrm{H} \%,>95)$ obtained at $-78^{\circ} \mathrm{C}$ was kept at $0^{\circ} \mathrm{C}$ in the presence of boron trifluoride etherate, depolymerization occurred and yielded DBO monomer until its concentration reached the equilibrium monomer concentration at $0^{\circ} \mathrm{C}$. The polymer recovered from this solution was found to possess nearly the same stereoregularity $(\mathrm{EQ}-\mathrm{H} \%, 76)$ as that of the polymer prepared in the polymerization in methylene chloride at $0^{\circ} \mathrm{C}$. The decrease in the stereoregularity of the recovered polymer may be partly due to the repolymerization of the monomer regenerated by the depolymerization, but rather it seems more reasonable to ascribe it to the inversion of the configuration through the acyclic oxonium ion VII.

In the discussion above, no role is attributed to the counter anions in the steric control of the polymerization of DBO, because all the experiments so far were carried out by using boron trifluoride etherate as a common initiator. However, it appears likely that counter anions play an important role in controlling the steric course of the cationic polymerization of $\mathrm{DBO}$, depending on characteristics such as sizes and polarizabilities.

In summary, the cationic ring-opening polymerization of DBO proceeds by an $S_{N} 2$ reaction between the cyclic trialkyloxonium ion $\mathbf{I}$ and the monomer, at least at lower temperatures, leading to the stereoregular polymer. The formation of the stereoirregular polymer at higher temperatures and in polar solvents may be ascribable to the participation of the carboxonium ion IV in the propagation reaction and/or to the occurrence of the oxonium ion-exchange reaction at the growing chain end. The contribution of the acyclic trialkyloxonium ion VII to the propagation reaction appears to be of minor importance, except in the late stage of the polymerization.

\section{REFERENCES}

1. H. Sumitomo, M. Okada, and Y. Hibino, $J$. Polym. Sci., Part B, 11, 871 (1972).

2. F. Sweet and R. K. Brown, Can. J. Chem., 46, 2289 (1968).

3. L. M. Jackman and S. Sternhell, "Application of Nuclear Magnetic Resonance Spectroscopy in Organic Chemistry", 2nd ed., Pergamon Press, New York, N.Y., 1969, p. 238.

4. P. Salomaa and A. Kankaanpera, Acta Chem. Scand., 15, 871 (1961).

5. M. Okada, Y. Yamashita, and Y. Ishii, Makromol. Chem., 80, 196 (1964).

6. P. Clasper and R. K. Brown, J. Org. Chem., 37, 3346 (1972). 


\section{Polymerization of 6,8-Dioxabicyclo[3.2.1]octane}

7. J. Zachoval and C. Schuerch, J. Amer. Chem. Soc., 91, 1165 (1969).

8. W. Gordy and S. C. Stanford, J. Chem. Phys., 7, 93 (1939); ibid., 8, 170 (1940); ibid., 10, 204 and 215 (1942).

9. S. Aoki, Y. Harita, T. Otsu, and M. Imoto, Bull. Chem. Soc. Japan, 38, 1922 and 1928 (1965).
10. T. Higashimura, T. Masuda, and S. Okamura, J. Polym. Sci., Part A-1, 71115 (1969).

11. R. Gumbs, S. Penczek, J. Jagur-Grodzinski, and M. Szwarc, Macromolecules, 2, 77 (1969).

12. M. Okada, H. Sumitomo, and Y. Hibino, unpublished data. 